\title{
Imaging Cells and Bionanoparticles by STEM and EFTEM Tomography
}

\author{
R.D. Leapman, M.A. Aronova, D.M. Cox, A. Adams, G. Zhang, A.A. Sousa
}

National Institute of Biomedical Imaging and Bioengineering, National Institutes of Health, Bethesda, MD 20892

Electron tomography (ET) based on bright-field transmission electron microscopy is a powerful technique for determining the three-dimensional structure of cells at the level of organelles and supramolecular assemblies [1]. Typically, amplitude contrast is used to perform conventional ET of fixed, embedded, stained and sectioned preparations, and phase contrast is used to perform cryo ET of cells rapidly frozen in a hydrated native state. For some applications, however, it is useful to perform ET using alternative contrast modes based on scanning transmission electron microscopy (STEM) [2-6], and energy filtered transmission electron microscopy (EFTEM) [7,8]. These techniques provide complementary information, as well as extend the thickness range that can be analyzed by ET. The techniques of STEM and EFTEM tomography can also be used to characterize a range of functionalized bionanoparticles and their interactions with cells in 3D.

The high collection efficiency of the annular dark-field detector in STEM tomography enables heavy-atom clusters (e.g., $\left.\mathrm{Au}_{67}\right)$ to be visualized directly in suitably thin specimens $(\sim 50 \mathrm{~nm})$ that are lightly stained [5]. Such nanoparticle labels can be conjugated with biological molecules such as antibody fragments to which fluorescent dyes can also be attached, e.g., Fluoronanogold (Nanoprobes Inc). This approach facilitates correlative ET/optical fluorescence microscopy of specific proteins, which can be localized in 3D. It is also possible to attach Nanogold labels and fluorescent dye labels to other molecules (e.g., peptides) in order to track their locations in cells at the nanoscale.

Acquisition of EFTEM tomographic tilt series at energy losses above and below the core edges of specific chemical elements can reveal the $3 \mathrm{D}$ distribution of biomolecules for which those elements serve as intrinsic labels. Thus, phosphorus reveals the distributions of nucleic acid, and nitrogen the distribution of protein plus nucleic acid [9]. Moreover, we have shown that the 3D elemental distributions can be quantified if the net core-edge signal is normalized by the zero-loss image and the corresponding core-edge scattering cross section [8]. It is important to employ an appropriate reconstruction scheme for the $3 \mathrm{D}$ reconstruction; in this regard, we have used the simultaneous iterative reconstruction (SIRT) algorithm, which minimizes the residual between the measured elemental distributions at different tilt angles and the corresponding projections through the reconstruction. The resulting technique of quantitative electron spectroscopic tomography (QuEST) has been applied to image nucleic acid within the cell nucleus. We have also shown that it is feasible to combine fluorescence optical imaging together with QuEST and STEM tomography to localize specific protein assemblies within the cell nucleus and to determine the environment of those proteins relative to chromatin [10].

Both EFTEM and STEM tomography can be combined to characterize the composition and 3D structure of composite bionanoparticles, such as $\mathrm{Au} / \mathrm{Fe}_{3} \mathrm{O}_{4}$ conjugated with peptides and fluorescent dyes [11]. We have also used STEM tomography to image small molecular clusters of cisplatin anticancer drug conjugated to bundles of single-walled carbon nanotubes that are introduced as a 
therapeutic agent into cancer cells [12].

By using EFTEM to select the most probable energy loss it is possible to perform ET on thick plastic sections while avoiding loss of spatial resolution due to chromatic aberration, but only at the cost of greatly reduced signal-to-noise ratio [13]. The absence of imaging lenses after the specimen when the electron microscope is operated in STEM mode has enabled us to perform electron tomography on micrometer-thick plastic embedded specimens without loss of spatial resolution. Furthermore, a narrow probe convergence angle of less than $2 \mathrm{mrad}$ minimizes geometrical beam broadening while maintaining a diffraction-limited resolution of approximately $1 \mathrm{~nm}$. We have found that an axial bright-field detector provides improved spatial resolution throughout the specimen thickness relative to an annular dark-field detector because axial detection excludes multiply scattered electrons that have broader spatial and angular distributions at the exit surface [14,15]. Monte Carlo simulations performed for a beam-energy of $300 \mathrm{keV}$ showed that a bright-field STEM detector of about 10mrad outer semi-angle offers a good compromise between reducing image blurring at the bottom of thick sections and maintaining a sufficiently high SNR for specimen features situated at the top surface [16].

We have demonstrated dual tilt-axis STEM tomography with optimized axial bight-field detector geometry by application to micrometer-thick sections of insulin-secreting beta cells from mouse pancreatic islet [16]. The quality of the resulting three-dimensional reconstructions was comparable to that obtained from much thinner (0.3-micrometer) sections using conventional TEM tomography. The increased range of specimen thickness accessible to axial STEM tomography without the need for serial sectioning enables the 3D visualization of more complex and larger subcellular structures. Future applications of STEM tomography to complex tissues such as neuronal circuitry appear to be very promising [17].

\section{References}

[1] V. Lucic, F. Förster, W. Baumeister, Annu. Rev. Biochem. 74 (2005) 833.

[2] P.A. Midgley, M. Weyland, Ultramicroscopy 96 (2003) 413.

[3] I. Arslan, J.R. Tong, P.A. Midgley, Ultramicroscopy 106 (2006) 994.

[4] A.E. Yakushevska et al., J. Struct. Biol. 159 (2007) 381.

[5] A.A. Sousa et al., J. Struct. Biol. 159 (2007) 507.

[6] N. de Jonge et al., Proc. Nat. Acad. Sci. USA 106 (2009) 2159.

[7] R.D. Leapman et al., Ultramicroscopy 100 (2004) 115.

[8] M.A. Aronova et al., J. Struct. Biol. 160 (2007) 35.

[9] D.P. Bazett-Jones, M.J. Hendzel, Methods Enzymol. 17 (1999) 188.

[10] M.A. Aronova et al., Microsc. Microanal. 15 (Suppl 2) (2009) 920.

[11] J. Xie et al., Curr. Med. Chem. 16 (2009) 1278.

[12] A. Bhirde et al., Nanomedicine 4 (2009) 763.

[13] J. C. Bouwer et al. J. Struct. Biol. 148, 297-306 (2004)

[14] A.A. Sousa et al., Ultramicroscopy 109 (2009) 213.

[15] M.F. Hohmann-Marriott, A.A. Sousa et al., Nature Methods 6 (2009) 729.

[16] A.A. Sousa et al., J. Struct. Biol. (2011) in press.

[17] This research was supported by the intramural program of the National Institute of Biomedical Imaging and Bioengineering, NIH. 